\title{
DISCOVERY OF A LOW-MASS COMPANION AROUND HR 3549
}

\author{
D. Mawet ${ }^{1,2,5}$, T. David ${ }^{1}$, M. Bottom ${ }^{1}$, S. Hinkley ${ }^{3}$, K. Stapelfeldt $^{4}$, D. PadgetT ${ }^{4}$, \\ B. Mennesson ${ }^{2}$, E. Serabyn ${ }^{2}$, F. Morales ${ }^{2}$, and J. Kunn ${ }^{2}$ \\ ${ }^{1}$ Department of Astronomy, California Institute of Technology, 1200 E. California Boulevard, MC 249-17, Pasadena, CA 91125, USA; dmawet@astro.caltech.edu \\ 2 Jet Propulsion Laboratory, California Institute of Technology, 4800 Oak Grove Drive, Pasadena, CA 91109, USA \\ ${ }^{3}$ Department of Physics and Astronomy, University of Exeter, Physics Building, Stocker Road, Exeter EX4 4QL, UK \\ ${ }^{4}$ NASA Goddard Space Flight Center, 8800 Greenbelt Road, Greenbelt, MD 20771, USA \\ Received 2015 July 1; accepted 2015 August 21; published 2015 September 28
}

\begin{abstract}
We report the discovery of a low-mass companion to HR 3549, an A0V star surrounded by a debris disk with a warm excess detected by WISE at $22 \mu \mathrm{m}$ (10 $\sigma$ significance). We imaged HR $3549 \mathrm{~B}$ in the L band with NAOSCONICA, the adaptive optics infrared camera of the Very Large Telescope, in January 2013 and confirmed its common proper motion in 2015 January. The companion is at a projected separation of $\simeq 80 \mathrm{AU}$ and position angle of $\simeq 157^{\circ}$, so it is orbiting well beyond the warm disk inner edge of $r>10 \mathrm{AU}$. Our age estimate for this system corresponds to a companion mass in the range $15-80 M_{J}$, spanning the brown dwarf regime, and so HR $3549 \mathrm{~B}$ is another recent addition to the growing list of brown dwarf desert objects with extreme mass ratios. The simultaneous presence of a warm disk and a brown dwarf around HR 3549 provides interesting empirical constraints on models of the formation of substellar companions.
\end{abstract}

Key words: brown dwarfs - instrumentation: adaptive optics - instrumentation: high angular resolution stars: imaging - stars: low-mass

\section{INTRODUCTION}

Although high contrast imaging of self-luminous exoplanets or brown dwarfs around young stars is already difficult because of the angular resolution and contrast required, a further complication is that the interpretation of the nature of any companions found in these images is made difficult because it relies upon theoretical models of formation and evolution which depend on a good knowledge of the host star characteristics. While instruments, observing strategies, and post-processing techniques have undeniably improved over the past decade, accurate stellar age determination is becoming the most pressing challenge of characterization. Because they are a robust yet imprecise sign of youth, it is no coincidence that the majority of exoplanets imaged by adaptive optics are found in systems with debris disks: HR 8799 (Marois et al. 2008), $\beta$ Pictoris (Lagrange et al. 2010), HD 95086 (Rameau et al. 2013), Fomalhaut (Kalas et al. 2008), GJ 758 (Thalmann et al. 2009), and HD 106906 (Bailey et al. 2014). We note that the GJ 504 system is, out of all bona fide directly imaged exoplanets, currently the only one without confirmed infrared excess, but is also the subject of an on-going controversy about its age. Fuhrmann \& Chini (2015) indeed suggest that the GJ 504 system is actually of Solar age, and that GJ 504b is thus likely not a $\sim 4$ Jupiter mass planet, but a 4.5 Gyr old Brown Dwarf.

Debris disks are the signposts of planetary systems. Collisions among asteroidal and cometary parent bodies maintain the observed dust population against losses to radiative forces. Since dust production is enhanced by gravitational stirring, debris disks systems are natural targets for giant planet imaging searches. It has been established both theoretically and observationally that warm dust is transient. Its presence thus serves as a marker for a young or dynamically

\footnotetext{
5 Based on observations made with ESO Telescopes at the La Silla Paranal Observatory under programs: 090.C-0486A and 094.C-0406A.
}

active planetesimal belt, and clearly indicates that the host star possesses some kind of planetary system. Like self-luminous exoplanets, the brightness of a debris disk decays with time. To first order, the magnitude of the warm excess serves as a chronometer: $22 / 24 \mu \mathrm{m}$ flux densities larger than $1.2 \times$ the stellar photosphere are almost always found in sources younger than $1 \mathrm{Gyr}$, and more typically, in stars with ages less than a few hundred Myr. This theoretical expectation has been borne out through both observational work and computational modeling. Rieke et al. (2005) have shown that warm excess, as observed at $24 \mu \mathrm{m}$, is a steeply declining function of stellar age in a sample of 266 A stars. Models of the dynamical evolution of planetesimal swarms confirm this behavior for A stars (Wyatt et al. 2007), punctuated by transient spikes of higher dust content following major planetesimal collisions.

Here we report the detection of a bound low-mass companion to dusty host star HR 3549. This discovery, reminiscent of $\kappa$ Andromedae (Carson et al. 2013; Hinkley et al. 2013), adds to the collection of extreme mass ratios substellar companions filling the brown dwarf desert.

\section{FUNDAMENTAL PARAMETERS OF HR 3549}

HR 3549 (HD 76346, HIP 43620) is a main-sequence A0 star of visual magnitude 6 (Table 1). The parallax measured by Hipparcos (van Leeuwen 2007) is $10.82 \pm 0.27$ mas, corresponding to a distance of $\simeq 92.5 \mathrm{pc}$. The WISE satellite measured $\mathrm{W} 1-\mathrm{W} 4=0.56 \pm 0.06 \mathrm{mag}$ of excess at $22 \mu \mathrm{m}$, and no excess at $12 \mu \mathrm{m}$ (Cutri et al. 2013; data from AllWISE cryogenic sky survey). For an A0 photosphere, this excess corresponds to a minimum $L_{\mathrm{dust}} / L_{\mathrm{star}}$ of $10^{-4}$, similar to the planet-bearing HR 8799. This value is for the case of a blackbody that peaks at $22 \mu \mathrm{m}$. The presence of any emission extended over a range of temperatures would add to this value.

We used the Bayesian Analysis for Nearby Young AssociatioNs II (BANYAN II) online tool to determine the membership probability of HR 3549 to nearby young 
Table 1

Properties of HR 3549

\begin{tabular}{|c|c|}
\hline Properties & Value \\
\hline Identifiers & 7 HR 3549, HD 76346, HIP 43620 \\
\hline Coord. (hms, dms) & $085303.77832-563858.1462$ \\
\hline Galactic coord. (deg) & $274.35-07.66$ \\
\hline Spectral type & $\mathrm{A} 0 \mathrm{~V}$ \\
\hline Distance (pc) & $92.5 \pm 2.5$ \\
\hline$V$ mag & 6.01 \\
\hline$L$ mag & $6.04 \pm 0.05$ \\
\hline$\mu_{\alpha} * \cos \delta\left(\operatorname{mas} \mathrm{yr}^{-1}\right)$ & $-22.81 \pm 0.26$ \\
\hline$\mu_{\delta}\left(\operatorname{mas~yr}^{-1}\right)$ & $36.54 \pm 0.26$ \\
\hline $\mathrm{RV}\left(\mathrm{km} \mathrm{s}^{-1}\right)$ & $23.90 \pm 2.2$ \\
\hline$[3.6]-[22] \mu \mathrm{m}$ & $0.56 \pm 0.06$ \\
\hline$T_{\text {eff }}(\mathrm{K})$ & $10207 \pm 347$ \\
\hline $\log (g)(\mathrm{cgs})$ & $4.20 \pm 0.14$ \\
\hline$v \sin i\left(\mathrm{~km} \mathrm{~s}^{-1}\right)$ & 212 (Glebocki \& Gnacinski 2005) \\
\hline Median Bayesian age (Myr) & 230 \\
\hline $68 \%$ age $\mathrm{CI}(\mathrm{Myr})$ & $120-360$ \\
\hline $95 \%$ age $\mathrm{CI}(\mathrm{Myr})$ & $10-390$ \\
\hline 2D interpolated age (Myr) & $200_{-160}^{+20}$ \\
\hline Median Bayesian mass $\left(M_{\odot}\right)$ & 2.32 \\
\hline $68 \%$ mass $\mathrm{CI}\left(M_{\odot}\right)$ & $2.2-2.5$ \\
\hline $95 \%$ mass $\mathrm{CI}\left(M_{\odot}\right)$ & $2.1-2.6$ \\
\hline
\end{tabular}

kinematic groups (Malo et al. 2013; Gagné et al. 2014). This tool is based on a comparison of Galactic position (XYZ) and space velocity (UVW) to well-defined moving groups closer than $100 \mathrm{pc}$ and younger than $200 \mathrm{Myr}$. No clear association can be found with any known nearby young moving group, so HR 3549 is likely a field A0 star.

Our direct interpolation in $\log \left(T_{\text {eff }}\right)-\log (g)$ space relative to PARSEC v1.1 evolutionary models (Bressan et al. 2012) yields $200 \mathrm{Myr}$, with a 40-220 Myr $68 \%$ confidence interval, determined from a distribution of 50,000 interpolated ages reached through Monte Carlo propagation of the associated errors in $\log \left(T_{\text {eff }}\right)-\log (g)$. However, as pointed out in David \& Hillenbrand (2015), direct interpolation does not account for the nonlinear mapping of time onto the H-R diagram, or the non-uniform distribution of stellar masses observed in the galaxy, and can lead to biases.

\section{OBSERVATIONS AND DATA REDUCTION}

We observed HR 3549 with NAOS-CONICA at the Very Large Telescope as part of program 090.C-0486(A), "L-band adaptive optics imaging of exoplanets around a sample of dusty A stars recently discovered by WISE' (PI: Mawet) on 2013 January 13 . We used the Lp-band filter. The L band (centered around $3.8 \mu \mathrm{m}$ ) is a compelling and competitive filter for ground-based planet surveys. This wavelength range offers significant advantages compared to shorter wavelengths: (i) the L-band contrast of planetary-mass companions with respect to their host stars is more favorable than in the $\mathrm{H}$ and $\mathrm{K}$ bands (Baraffe et al. 2003) so that lower-mass objects can be probed; and (ii) longer wavelengths lead to better image quality and a more stable point-spread function (PSF), with Strehl ratios well above $70 \%$. These advantages outweigh the increased sky background in the thermal infrared and the loss in resolution. Finally, we note that background star contamination probability rates will be near-zero at small separations in the $\mathrm{L}$ band, so minimal follow-up time will be needed to confirm candidates.
The data were acquired as a sequence of 10 exposures on a 5-point dithering pattern with offsets of about 6 arcsec each. Each exposure was the average of 100 frames with $0.25 \mathrm{~s}$ integration time, making for a total open shutter time of $250 \mathrm{~s}$. We used the pupil tracking mode where the instrument corotates with the telescope pupil to fix diffraction and speckles to the detector reference frame, allowing the sky to counter-rotate with the parallactic angle, effectively enabling angular differential imaging (ADI; Marois et al. 2006). ADI requires sufficient sky rotation to avoid self-subtraction of the companion signal at small angles, often leading to long sequences. Our strategy to overcome this limitation of ADI has been to limit the sequence duration and rely on our uniform target sample to build a library of reference PSFs, and perform reference star differential imaging. Indeed, if the sample is uniform in brightness, covers a reasonable range of observing conditions, and is spread uniformly across the sky, then correlated speckle patterns can be retrieved in the library of PSFs.

We reduced the data by subtracting a background made out of median-combined dithered frames, dividing by a flat field and interpolating for bad pixels and other cosmetics. The reduced images were then processed by two independent speckle calibration packages based on principal component analysis (Soummer et al. 2012), and the library of reference PSF for the speckle calibration.

\section{DISCOVERY OF A CANDIDATE LOW-MASS COMPANION TO HR 3549}

The 2013 January 13 data set showed a point source at a separation of $\simeq 0$." 9 and position angle of $\simeq 157^{\circ}$. The detection is unambiguous with a signal-to-noise ratio $(\mathrm{S} / \mathrm{N})$ of $\simeq 6$ (see Figure 1, left). The TRILEGAL starcount model (Girardi et al. 2012) estimates a probability of $7 \times 10^{-4}$ that it is an unrelated background object. While this number must be interpreted with caution, it is significant enough to warrant follow-up observations.

We acquired the second epoch data set as part of program 094.C-0406(A), "L-band adaptive optics imaging of exoplanets around a sample of dusty stars recently discovered by WISE. Part III: candidate follow-ups" (PI: Mawet), on 2015 January 14 , following a similar strategy as for the discovery epoch. The candidate was detected at roughly the same location though with a lower $S / N(\simeq 3$, see Figure 1 , right). The lower $S / N$ appears to be mostly due to the characteristics of the Aladdin2 detector installed in CONICA in 2015 January to replace the faulty Aladdin3 detector, and the additional thermal background induced by the different shielding characteristics of this detector configuration.

\section{ASTROMETRY AND COMMON PROPER MOTION ANALYSIS}

We performed astrometric and photometric measurement of both epochs using forward modeling of the off-axis companion point spread function with a Monte-Carlo Markov chain (MCMC) sampler in the $\alpha, \delta$, flux space. The MCMC sampler model also takes into account variable error bars, meaning that systematic under-reporting of errors should not affect the final result.

The final error bars on the astrometry were then conservatively set to the quadratic sum of the MCMC-derived error 

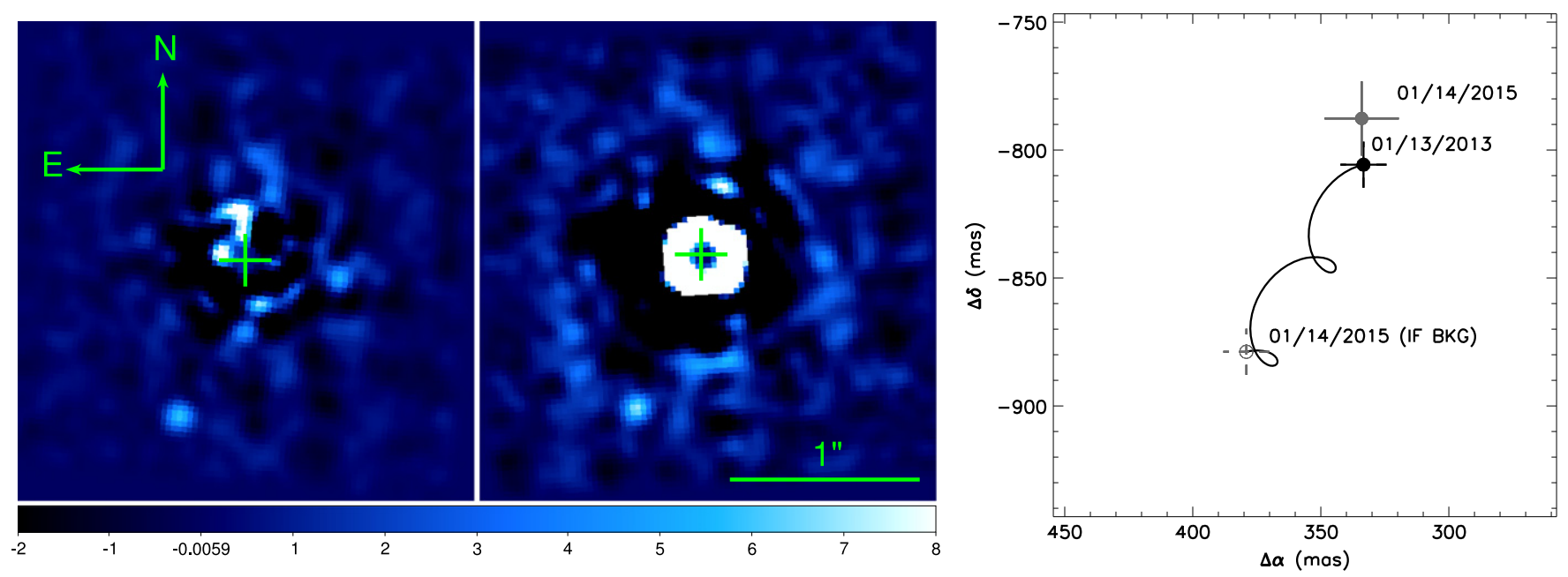

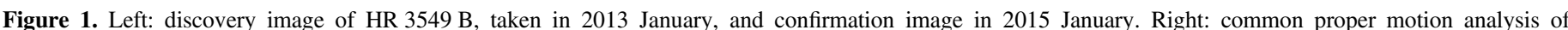

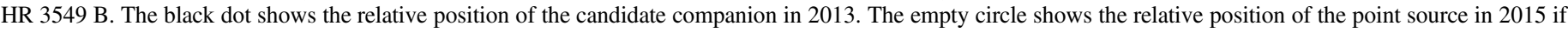
it were a background object, accounting for parallactic motion (plain curve). The gray dot is the measured position of the bound companion in 2015 .

Table 2

Astrometry and Photometry of the Low-mass Companion to HR 3549

\begin{tabular}{|c|c|c|c|c|c|c|}
\hline Data Set & Filter & $\mathrm{S} / \mathrm{N}$ & $\Delta \alpha\left({ }^{\prime \prime}\right)$ & $\Delta \delta\left({ }^{\prime \prime}\right)$ & $m$ & $M^{\mathrm{a}}$ \\
\hline NACO 2013 & $\mathrm{Lp}$ & $\simeq 6.25$ & $0.1333 \pm 0.009$ & $-0 . " 806 \pm 0$ ".009 & $13.85 \pm 0.25$ & $9.03 \pm 0.26$ \\
\hline NACO 2015 & Lp & $\simeq 3.1$ & $0 . " 334 \pm 0$ ". 015 & -0 ". $788 \pm 0$ " 015 & $13.63 \pm 0.5$ & $8.5 \pm 0.505$ \\
\hline
\end{tabular}

bars, which can still be biased by underlying speckle noise, and the empirically derived influence of $\mathrm{S} / \mathrm{N}$ on $\alpha, \delta$, flux. Indeed, astrometric precision (Guyon et al. 2012) is proportional to $\mathrm{FWHM} /(2 \times \mathrm{S} / \mathrm{N})$ in the speckle-noise dominated regime, where FWHM is the full width at half maximum of the resolution element $\lambda / D$, with observing wavelength $\lambda$ and telescope diameter $D$.

As far as the astrometric calibration of CONICA is concerned, we used the $27.1 \pm 0.04$ mas pixel $^{-1}$ plate scale and $-0.45 \pm 0.09$ true north offset of Absil et al. (2013) for the 2014 epoch, and the $26.99 \pm 0.02$ mas pixel $^{-1}$ plate scale and $+0.31 \pm 0.02$ true north offset for the 2015 epoch, measured after the NACO recommissioning with the Aladdin2 chip on UT1 (personal communication from NACO instrument scientist Julien Girard, European Southern Observatory). These additional systematic offsets and corresponding error bars were folded into both epoch astrometric positions and associated error bars (Table 2) to perform the common proper motion analysis (CPM) shown in Figure 1 (right). Following the CPM analysis, we determined that the probability of the discovered candidate to be a background object is $2 \times 10^{-8}$.

\section{AGE OF HR 3549}

As mentioned earlier, direct interpolation in an H-R diagram or color-magnitude diagram can lead to biases in derived ages. Following David \& Hillenbrand (2015), we thus used a Bayesian analysis of the star's location in $\log T_{\text {eff }}-\log g$ space relative to solar metallicity $(Z=0.015)$ PARSEC v1.1 evolutionary models (Bressan et al. 2012), yielding a 68\% age confidence interval of $120-360 \mathrm{Myr}$, which is consistent with the average age of A0 field dwarfs. We performed likelihood calculations on a $1000 \times 1000$ grid from $1 \mathrm{Myr}$ to $10 \mathrm{Gyr}$ in age, and $1-10 M_{\odot}$ in mass. We used the Salpeter IMF (Salpeter $1955)$ as the prior on stellar mass and a uniform prior in linear age (i.e., constant star formation rate). The atmospheric parameters are determined from combination of $u v b y \beta$ photometry and ATLAS9 models (Castelli \& Kurucz 2004, 2006), and the details of both the atmospheric characterization and age determination are presented in David \& Hillenbrand (2015, hereafter DH15).

Intermediate-mass stars are rapid rotators, and the effect of this rotation is to make the star appear cooler, more luminous, and hence older than a non-rotating star of the same mass. Additionally, rapid rotators spend a longer time on the main sequence than slow rotators. Recently, DH15 and Brandt \& Huang (2015, hereafter BH15) have shown that the rotational effects on the inferred ages of intermediate-mass stars are substantial. $^{6}$

Consequently, we explored two possible solutions for the age of HR 3549. The first solution is based on the rotationcorrected atmospheric parameters derived in DH15, $T_{\text {eff }}=11176 \pm 380 \mathrm{~K}, \log g=4.33 \pm 0.14 \mathrm{dex}$ (see Figure 2). At this position, direct interpolation yields an age of $43_{-37}^{+82} \mathrm{Myr}$, which is consistent with a previous estimate of 55 Myr (Westin 1985). ${ }^{7}$ The Bayesian age analysis in this case yields a median age of $110 \mathrm{Myr}$ with $68 \%$ and $95 \%$ confidence intervals of 10-150, 10-250 Myr, respectively.

The second, more likely, solution is based on the uncorrected atmospheric parameters from DH15, listed in Table 1. This set of atmospheric parameters yields a median Bayesian age and $68 \%$ confidence interval of $230_{-110}^{+130} \mathrm{Myr}$, with a corresponding interpolated age of $200_{-160}^{+20} \mathrm{Myr}$ (where the uncertainties are

\footnotetext{
6 We note these authors account for rotation in different ways when determining ages for such stars. While DH15 apply the corrective formulae of Figueras \& Blasi (1998) to the atmospheric parameters prior to age determination (assuming $v \sin i \approx v_{\text {rot }}$ ), BH15 marginalize over projected rotational velocity.

7 Westin (1985) discusses age errors in a general sense but does not provide information for assigning errors to the A0 stars in that sample.
} 

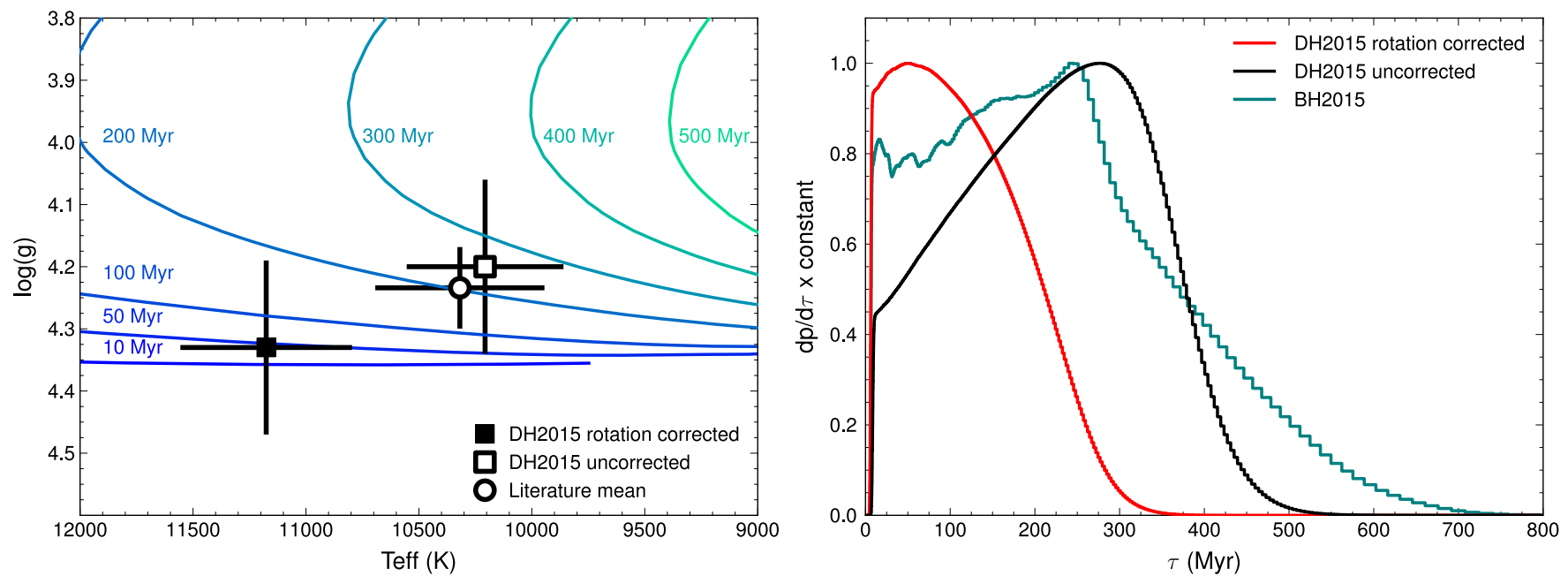

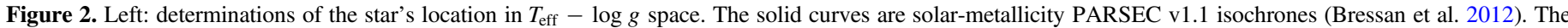

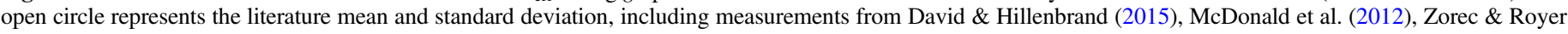

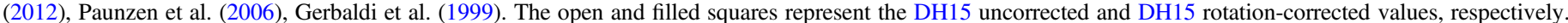

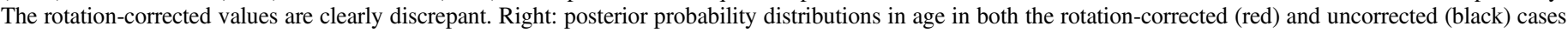
from DH15. The teal curve is the age posterior from Brandt \& Huang (2015). Taken collectively, the age of HR 3549 is in the range of 50-400 Myr.

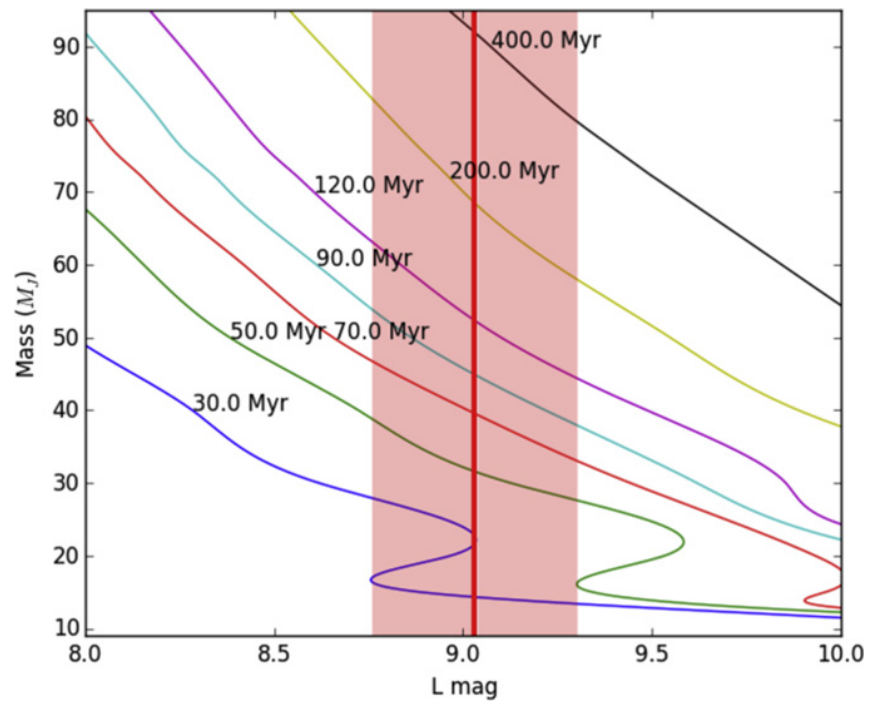

Figure 3. Mass $\left(M_{J}\right)$ vs. Luminosity ( $L$ mag) diagram for the evolutionary model BT-Settl. HR 3549 B's L-band photometry and associated uncertainty is in shaded red.

determined from Monte Carlo error propagation). We consider this solution more likely for the following reasons: (1) the effective temperature is more consistent with modern spectral type scales which suggest $T_{\text {eff }} \approx 9700 \mathrm{~K}$ at A0 (Adelman 2004; Pecaut \& Mamajek 2013), (2) both $T_{\text {eff }}$ and $\log g$ in this case are consistent with the mean of previous determinations from the literature, (3) this older age is more consistent with the average age of A0 field dwarfs, (4) as noted in Section 2, the star is unlikely to be associated with known young moving groups, (5) there is some evidence presented in DH15 that the procedure used to determine the rotation-corrected parameters is over-aggressive, (6) there are no other significant indicators of youth for HR 3549, and (7) the older age is consistent with other modern estimates: $300 \pm 51 \mathrm{Myr}$ (Zorec \& Royer 2012) and $138 \pm 98 \mathrm{Myr}$ (Gerbaldi et al. 1999), both from H-R diagram analyses.
Figure 2 (left) demonstrates the position of HR 3549 in $\log T_{\text {eff }}-\log g$ space, relative to evolutionary models. The difficulty of age-dating intermediate-mass stars on and near the main sequence is evident from the typically large uncertainties in surface gravity (or equivalently, luminosity). The effect of including rotation is also illustrated by the significantly different atmospheric parameters obtained.

Figure 2 (right) shows the posterior probability distribution function (PDF) in age for HR 3549, originally derived in DH15. Also depicted is a PDF derived from the similar Bayesian approach to isochrone age-dating of Brandt \& Huang (2015). The BH15 PDF was generated assuming a Gaussian prior on $[\mathrm{Fe} / \mathrm{H}]$, with $\mu=-0.1$ and $\sigma=0.2 \mathrm{dex}$, consistent with the distribution observed for intermediate-mass stars in the solar neighborhood. ${ }^{8}$ The BH15 PDF is broader than that of DH15 due to the fact that those authors marginalize over mass, metallicity, rotational velocity, and inclination, three parameters which can substantially affect the inferred ages of intermediate-mass dwarfs. In contrast, DH15 marginalize over mass only, while the typically minor differences in metallicity are implicitly accounted for in the atmospheric parameter uncertainties. For comparison, the BH15 PDF yields a median age of $220 \mathrm{Myr}$, with $68 \%$ and $95 \%$ confidence intervals of 10-290, 10-500 Myr, respectively.

All estimates suggest the age is $<500 \mathrm{Myr}$, and notably the three most recent estimates suggest $\tau>200 \mathrm{Myr}$, which is consistent with the average age of A0 field dwarfs. The range of published ages illustrates the difficulty of age-dating on and near the main sequence, particularly for intermediate-mass stars for which empirical age-dating methods are either non-existent or uncalibrated. We adopt the median Bayesian age and 68\% confidence interval as the final age and uncertainties for HR 3549, $\tau \approx 230_{-110}^{+130}$ Myr David \& Hillenbrand (2015). However, the literature range can also be used to infer the loosest reasonable constraints on the system age: 50-400 Myr. For completeness, we consider this broad range of plausible ages when inferring the companion mass.

8 The BH15 PDF was downloaded from http://bayesianstellarparameters.info/ 


\section{PHOTOMETRY AND PROPERTIES OF THE COMPANION}

The forward modeling MCMC approach to measure the companion photometry described above yields an absolute $\mathrm{L}$ magnitude of $9.03 \pm 0.26$ for the companion, including parallax/distance and L-band magnitude uncertainties. To infer the companion properties, we used the BT-Settl evolutionary model (Allard 2014) and our adopted conservative age range of 50-400 Myr. BT-Settl covers the range from solar-mass stars to the latest-type $\mathrm{T}$ and $\mathrm{Y}$ dwarfs, and reproduces the formation of clouds and in particular their clearing at the $\mathrm{L} / \mathrm{T}$ transition. We derived a mass range of 15-90 $M_{J}$ (Figure 3), and effective temperature between 1900 and $2700 \mathrm{~K}$, placing HR $3549 \mathrm{~B}$ in the L-dwarf regime.

\section{DISCUSSION}

No far-infrared observations of HR 3549 are listed in the data archives for the Spitzer Space Telescope or Herschel Space Observatory, so the properties of its debris disk can only be estimated from the WISE survey data. The AllWISE survey magnitudes are $6.04 \pm 0.05,5.97 \pm 0.04,6.06 \pm 0.02$, and 5.50 \pm 0.03 - corresponding to 3.6, 4.5, 11.8, and $22.0 \mu \mathrm{m}$, respectively. An excess of $0.56 \pm 0.06 \mathrm{mag}$ is detected at $22 \mu \mathrm{m}$, but no excess is evident at shorter wavelengths. Using a $3 \sigma$ upper limit to the $11.8 \mu \mathrm{m}$ excess $(0.05 \mathrm{mag}$ ), and assuming blackbody emission, the upper limit to the disk dust temperature is $168 \mathrm{~K}$. For grain sizes of a few microns, this corresponds to a disk inner edge of $r>10 \mathrm{AU}$.

While the WISE data provide no constraint on the disk outer radius, HR $3549 \mathrm{~B}$ appears to be massive enough to gravitationally clear any disk material in its vicinity. It is unlikely that any disk material located beyond the companion's $>80 \mathrm{AU}$ orbital separation would be warm enough $(\sim 120 \mathrm{~K})$ to emit significantly at $22 \mu \mathrm{m}$. The most likely scenario is therefore that HR 3549 B orbits exterior to a warm dust belt that is the source of the WISE excess. An exterior cold dust belt beyond HR 3549 B orbiting radius may also exist but would require ALMA observations to detect.

The HR 3549 system shares several features with the $\kappa$ Andromedae system (Carson et al. 2013; Hinkley et al. 2013). Like $\kappa$ Andromedae, HR 3549 is a late-B/early-A type star with a mass $\sim 3 M_{\odot}$, and a poorly constrained age. Further, the companions in both systems are "brown dwarf desert" objects with masses in the range of $\sim 15-80 M_{\mathrm{Jup}}$, corresponding to mass ratios of $\sim 1 \%$. Such "extreme mass ratio systems" (e.g., Hinkley et al. 2015b), are particularly important for constraining the formation mechanisms of stars in this mass range. Indeed, several works (e.g., Delgado-Donate et al. 2004; Kouwenhoven et al. 2007) have suggested that the multiplicity of intermediate-mass stars may serve as a reservoir for the conserved initial angular momentum in the protostellar cloud.

Lastly, we note that no other companion more massive or similar to HR 3549 B is detected around HR 3549 from the effective inner working angle of $\simeq 0$ "! 3 (projected separation of $\simeq 30 \mathrm{AU}$ ) to the outer edge of the effective field of view of $\simeq 12^{\prime \prime}(\simeq 1000 \mathrm{AU})$.

\section{CONCLUSION}

This paper presented the detection of a substellar companion orbiting at a projected separation of $\simeq 80$ AU around HR 3549, a disk-bearing A0V star. The characterization of the companion is made difficult by the uncertain age determination for field $\mathrm{A}$ stars. Spectroscopic follow-up using medium resolution slit spectroscopy is the next step to further characterize the low-mass object around HR 3549 (Hinkley et al. 2015a). Indeed spectral indices to quantitatively measure the strength of the $\mathrm{FeH}, \mathrm{VO}$, and KI spectral features have been demonstrated to be robust age markers, but require medium-resolution spectroscopy $(R>300)$.

The first author is grateful to Prof. Lynne Hillenbrand (Caltech) and Ben Zuckerman (UCLA) for their advice and comments. T.J.D is supported by the National Science Foundation Graduate Research Fellowship under grant No. DGE1144469. M.B. is supported by a NASA Space Technology Research fellowship, grant No. NNX13AN42H. This publication makes use of data products from the Wide-field Infrared Survey Explorer, which is a joint project of the University of California, Los Angeles, and the Jet Propulsion Laboratory/California Institute of Technology, funded by the National Aeronautics and Space Administration.

Facility: Very Large Telescope.

\section{REFERENCES}

Absil, O., Milli, J., Mawet, D., et al. 2013, A\&A, 559, L12

Adelman, S. J. 2004, in IAU Symp. 224, The A-Star Puzzle, ed. J. Zverko et al. (Cambridge: Cambridge Univ. Press), 1

Allard, F. 2014, in IAU Symp. 299, Exploring the Formation and Evolution of Planetary Systems, ed. M. Booth et al. (Cambridge: Cambridge Univ. Press), 271

Bailey, V., Meshkat, T., Reiter, M., et al. 2014, ApJL, 780, L4

Baraffe, I., Chabrier, G., Barman, T. S., Allard, F., \& Hauschildt, P. H. 2003, A\&A, 402, 701

Brandt, T. D., \& Huang, C. X. 2015, ApJ, 807, 58

Bressan, A., Marigo, P., Girardi, L., et al. 2012, MNRAS, 427, 127

Carson, J., Thalmann, C., Janson, M., et al. 2013, ApJL, 763, L32

Castelli, F., \& Kurucz, R. L. 2004, arXiv:astro-ph/0405087

Castelli, F., \& Kurucz, R. L. 2006, A\&A, 454, 333

Cutri, R. M., Wright, E. L., Conrow, T., et al. 2013, yCat, 2328, 0

David, T. J., \& Hillenbrand, L. A. 2015, ApJ, 804, 146

Delgado-Donate, E. J., Clarke, C. J., Bate, M. R., \& Hodgkin, S. T. 2004, MNRAS, 351, 617

Figueras, F., \& Blasi, F. 1998, A\&A, 329, 957

Fuhrmann, K., \& Chini, R. 2015, ApJ, 806, 163

Gagné, J., Lafrenière, D., Doyon, R., Malo, L., \& Artigau, É 2014, ApJ, 783, 121

Gerbaldi, M., Faraggiana, R., Burnage, R., et al. 1999, A\&AS, 137, 273

Girardi, L., Barbieri, M., Groenewegen, M. A. T., et al. 2012, in TRILEGAL, a TRIdimensional ModeL of thE GALaxy: Status and Future, ed. A. Miglio, J. Montalbán \& A. Noels (Berlin: Springer), 165

Glebocki, R., \& Gnacinski, P. 2005, yCat, 3244, 0

Guyon, O., Bendek, E. A., Eisner, J. A., et al. 2012, ApJS, 200, 11

Hinkley, S., Bowler, B. P., Vigan, A., et al. 2015a, ApJL, 805, L10

Hinkley, S., Kraus, A. L., Ireland, M. J., et al. 2015b, ApJL, 806, L9

Hinkley, S., Pueyo, L., Faherty, J. K., et al. 2013, ApJ, 779, 153

Kalas, P., Graham, J. R., Chiang, E., et al. 2008, Sci, 322, 1345

Kouwenhoven, M. B. N., Brown, A. G. A., Portegies Zwart, S. F., \& Kaper, L. 2007, A\&A, 474, 77

Lagrange, A.-M., Bonnefoy, M., Chauvin, G., et al. 2010, Sci, 329, 57

Malo, L., Doyon, R., Lafrenière, D., et al. 2013, ApJ, 762, 88

Marois, C., Lafrenière, D., Doyon, R., Macintosh, B., \& Nadeau, D. 2006, ApJ, 641, 556

Marois, C., Macintosh, B., Barman, T., et al. 2008, Sci, 322, 1348

McDonald, I., Zijlstra, A. A., \& Boyer, M. L. 2012, MNRAS, 427, 343

Paunzen, E., Schnell, A., \& Maitzen, H. M. 2006, A\&A, 458, 293

Pecaut, M. J., \& Mamajek, E. E. 2013, ApJS, 208, 9

Rameau, J., Chauvin, G., Lagrange, A. M., et al. 2013, ApJL, 779, L26

Rieke, G. H., Su, K. Y. L., Stansberry, J. A., et al. 2005, ApJ, 620, 1010

Salpeter, E. E. 1955, ApJ, 121, 161

Soummer, R., Pueyo, L., \& Larkin, J. 2012, ApJL, 755, L28

Thalmann, C., Carson, J., Janson, M., et al. 2009, ApJL, 707, L123

van Leeuwen, F. 2007, A\&A, 474, 653

Westin, T. N. G. 1985, A\&AS, 60, 99

Wyatt, M. C., Smith, R., Su, K. Y. L., et al. 2007, ApJ, 663, 365

Zorec, J., \& Royer, F. 2012, A\&A, 537, A120 